Acta Crystallographica Section D

Biological

Crystallography

ISSN 0907-4449

\section{Ardeschir Vahedi-Faridi, ${ }^{a} \neq$ Vivian Stojanoff ${ }^{b} \neq$ and Joanne I. Yeh $^{\mathrm{a} *}$}

aBrown University, Department of Molecular Biology, Cell Biology and Biochemistry, 70 Ship Street, Providence, RI 02903, USA, and

${ }^{\mathbf{b}}$ National Synchrotron Light Source, Upton, NY 11973-5000, USA

₹ These authors contributed equally to this work.
(C) 2005 International Union of Crystallography Printed in Denmark - all rights reserved

\title{
The effects of flash-annealing on glycerol kinase crystals
}

Reflection profiles from glycerol kinase crystals were analyzed to determine the effect of flash-cooling on mosaicity $(\eta)$ and peak intensity in order to reveal changes in mosaic domain structure and composition. The results showed that repeated flash-annealing causes a significant decrease in the averaged mosaicity along with an increase in the overall peak counts of reflections and an enhanced signal-to-noise ratio. Individual reflection-profile analysis revealed a mostly dual domain structure, showing the minimization of one domain as a result of flash-annealing.

\section{Introduction}

Within the last ten years, protein crystals have been routinely flash-cooled to avoid crystal degradation as a result of radiation damage in the X-ray beam (Rodgers, 1994, 1997; Garman \& Schneider, 1997). A disadvantage of this technique is the increase in crystal mosaicity that is frequently observed (Teng \& Moffat, 1998; Kriminski et al., 2002) and in many cases may lead to undesirable reflection overlap. Crystal mosaicity was first explained by Darwin (1922), who proposed a model which described crystals as composed of various mosaic blocks with differences in orientation, unit-cell size and lattice variations. Helliwell (1988) provided a theoretical analysis of what the smallest mosaicity in a protein crystal would be if it were perfect. Nave (1998) and Boggon et al. (2000) discussed how minute differences in orientation and unit-cell size of these blocks contribute to the overall increase in mosaicity. Teng \& Moffat (1998) showed that flash-cooling results in an initial extreme temperature gradient between the surface and the interior of the crystal. They speculated that this was a consequence of incomplete relaxation of the crystal lattice, consequently causing variations in the lattice which are exhibited in unit-cell dimension changes.

The possibility of improving crystal order by changing the rate at which crystals reach cryo-temperatures led to modifications and testing of various cooling protocols. These included cooling at different temperatures or the use of additional intermediary temperature steps (Teng \& Moffat, 1998) and correlating these with conformational and lattice relaxation times (Parak et al., 1987). Mitchell \& Garman (1994) suggested that optimizing the cryoprotectant concentration could minimize the mosaicity of cryocooled crystals. They found that there was a definite optimal cryoprotectant reagent concentration which corresponded to the highest resolution of diffraction and the minimum mosaicity and that these last two parameters were highly correlated with one another. Other modifications included altering or changing
Received 9 September 2004 Accepted 22 April 2005 
cryoprotectants to modify the glass-transition temperature, as initially proposed by Petsko (1975). These and other reports led to the idea that reduction of lattice stress through thawing of already cooled crystals may be possible via brief warming and re-cooling of crystals. The temporary application of 'heat' through thawing and subsequent relief of lattice constraints could then lead to a more ordered and homogenous distribution of unit cells upon re-cooling.

To test this idea, the technique of flash-annealing (FA) was developed. This technique, reported by Yeh \& Hol (1998), demonstrated that rapid thawing and recooling of crystals of glycerol kinase, while mounted on the goniometer head under a cold nitrogen stream at a temperature of $100 \mathrm{~K}$ (hence also referred to as 'in-situ annealing'), can dramatically improve diffraction quality. Flash-annealing decreased the overall mosaicity of glycerol kinase crystals by over $1^{\circ}$, as indicated by the mosaicity value given by the program $D E N Z O$. As defined in $D E N Z O$, mosaicity is the rocking angle in degrees in both the vertical and the horizontal directions which would generate all the spots seen on a still diffraction photograph. It includes contributions from X-ray bandwidth and beam crossfire (Otwinowski \& Minor, 2001). DENZO assumes a cosine model of the mosaicity function, approximately fullwidth at $5 \%$ maximum. However, the formula does not explain why the model works well, owing to (anti)correlations of errors (Otwinowski, 2005). In addition to lowering the overall mosaicity of glycerol kinase crystals, flash-annealing increased the resolution of the same crystals by $0.5 \AA$ (Yeh \& Hol, 1998).

An alternative annealing technique, referred to as macromolecular crystal annealing (MCA), was reported by Harp et al. (1998). In this approach, the crystal is removed from the coldstream, thawed in cryoprotectant and subsequently cryocooled again. MCA was first applied to nucleosome core particle crystals, where these crystals were removed from the cryostream and transferred to a large drop of cryoprotectant. The crystal remained intact and transparent during a subsequent flash-cooling step. Additional studies by Harp et al. (1998) on nucleosome core particles, histone octamers and lysozyme showed this procedure to be especially effective in rectifying problems such as ice rings resulting from mishandling of crystals during cooling. MCA improved the mosaicity by more than twofold for the nucleosome core particle, reducing this value from 0.825 to $0.345^{\circ}$. The histone octamer showed a more modest improvement of approximately $20 \%$ from 0.338 to $0.217^{\circ}$. The study indicated that the volume of the drop $(\sim 0.3 \mathrm{ml})$ was a key parameter, in addition to an incubation time of approximately $3 \mathrm{~min}$. The exact reasons for these particular volume and time parameters were not elaborated, although the authors concluded that the warming that resulted from crystal annealing allowed the reordering of misaligned mosaic blocks in the crystal, contributing to a lowering of the mosaic spread of the reflections. Interestingly, when the initial glycerol kinase crystals were annealed via the MCA approach, the crystals no longer diffracted upon recooling. It is possible that optimization of the MCA annealing parameters would have resulted in better annealing results; however, this method was not pursued since flash-annealing significantly improved diffraction quality (Yeh \& Hol, 1998).

In 1999, Harp and coworkers published a study comparing the flash-annealing technique with MCA. These techniques were applied to six different types of protein crystals (myoglobin, lysozyme, proteinase $\mathrm{K}$, concanavalin $\mathrm{A}$, nucleosome core particle and chicken histone octamer). Overall, they found that MCA was successful in five cases, whereas flash-annealing improved the crystal quality in four cases. It was speculated that the differences resulting from the application of the two techniques could be attributed to the effect of the crystal size on the warming time, as larger crystals require a longer thawing period for the crystals to 'clear up' (Harp et al., 1999).

Further studies were carried out, such as the work of Samygina et al. (2000), who employed a combination of increased salt concentration with crystal annealing using Escherichia coli pyrophosphatase and other protein crystals. Repeated cycles of annealing resulted in a dramatic improvement of the maximum resolution from 1.8 to $1.2 \AA$, along with a threefold to fivefold decrease in mosaicity. Ellis et

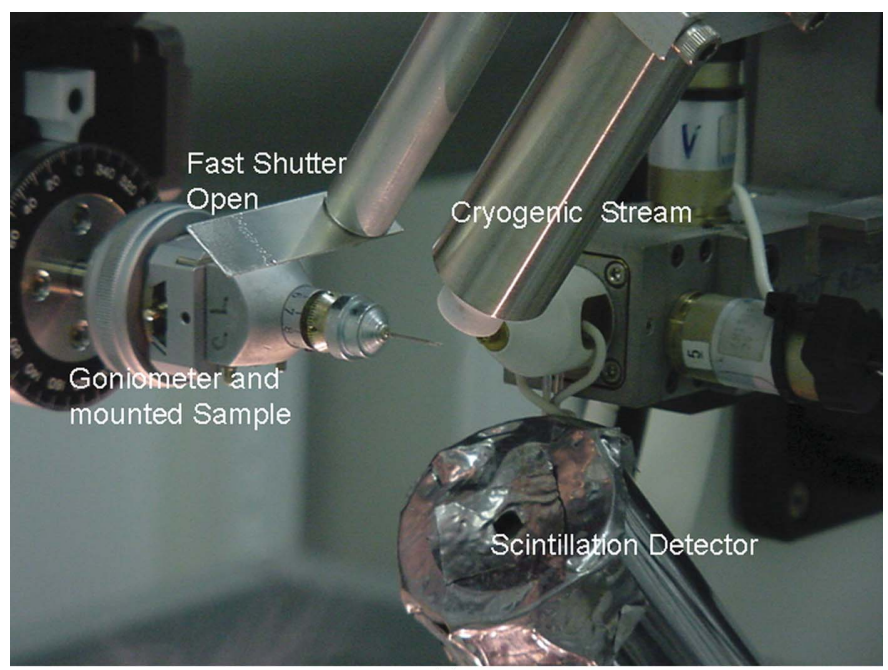

(a)

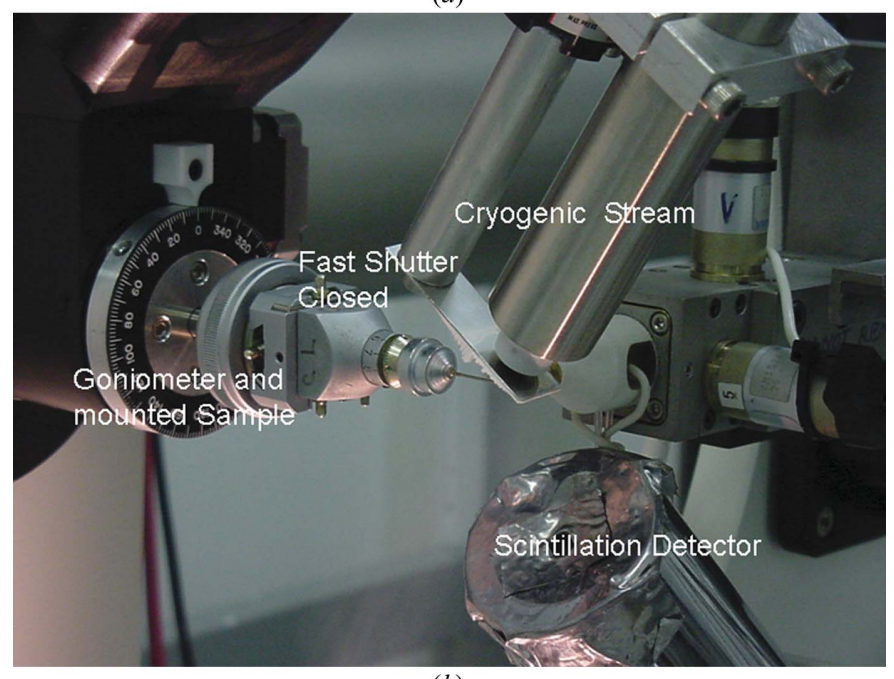

(b)

Figure 1

Image of the Cryo-Shutter in the $(a)$ open and $(b)$ closed state. 
Table 1

Diffraction and crystal mosaicity statistics for glycerol kinase crystals.

\begin{tabular}{|c|c|c|c|c|c|}
\hline Crystal & $\begin{array}{l}\text { Highest resolution } \\
\text { reflection } \\
\text { measured }(\AA)\end{array}$ & $\begin{array}{l}\eta_{\text {avg }} \\
\text { FWHM } \dagger\left(^{\circ}\right)\end{array}$ & $\begin{array}{l}\eta_{\text {avg }} \\
\text { FWQM } \dagger\left(^{\circ}\right)\end{array}$ & $\begin{array}{l}\text { No. of reflections } \\
\text { analyzed }\end{array}$ & $\begin{array}{l}\text { Average max. } \\
\text { peak intensity } \\
\text { (counts) }\end{array}$ \\
\hline \multicolumn{6}{|c|}{ Cryocrystals } \\
\hline cr_xtl1 & 2.23 & $0.232(0.062)$ & $0.392(0.125)$ & 564 & 766 \\
\hline cr_xtl2 & 2.55 & $0.253(0.068)$ & $0.448(0.117)$ & 286 & 1756 \\
\hline cr_xtl3 & 2.35 & $0.225(0.034)$ & $0.312(0.045)$ & 252 & 1152 \\
\hline \multicolumn{6}{|c|}{ Flash-annealed crystals } \\
\hline an_xtl1 & 2.19 & $0.171(0.039)$ & $0.280(0.072)$ & 774 & 656 \\
\hline an_xtl2 & 2.37 & $0.155(0.043)$ & $0.256(0.072)$ & 343 & 1851 \\
\hline an_xtl3 & 2.21 & $0.139(0.045)$ & $0.243(0.066)$ & 300 & 1030 \\
\hline
\end{tabular}

$\dagger$ Standard deviation is given in parentheses.

al. (2002) reported one of the most dramatic improvements in crystal quality using the flash-annealing technique. Crystals of both native and mutant copper nitrite reductase protein were studied. The crystals were annealed by blocking the coldstream for 10-15 s. It was found that the resolution limit was extended from 2.5 to $1.1 \AA$, with a concomitant decrease in mosaic spread from 1.5 to $0.3^{\circ}$. Kriminski et al. (2002) studied flash-cooling-induced disorder and annealing using tetragonal and triclinic hen egg-white lysozyme crystals. They speculated that crystal annealing reduces the distribution of lattice spacings and improves diffraction resolution. They similarly concluded that annealing produces larger and more homogeneous domains.

More recently, it was proposed that the solvent region plays an important role in the successful application of annealing techniques. Parkin \& Hope (2003) investigated the effect of crystal annealing on the solvent channels and structure of concanavalin A crystals. They found only minimal changes in the protein structure and the immediate hydration shell as a result of crystal annealing. The secondary disordered solvent region, however, showed some dramatic changes. Juers \& Matthews (2004) examined the role of solvent transport during annealing of $\beta$-galactosidase crystals. The crystals were flash-annealed with a blocking time of 2-3 s. The annealing process was repeated in cycles and the crystals showed a continuous decrease in overall mosaicity along with an improvement in $I / \sigma(I)$ per annealing cycle. These investigators postulated that the key to understanding crystal annealing is the role of the bulk solvent and the cryoprotectant concentration. An initially high cryoprotectant concentration will result in a net flow of condensed water into the crystal during the thawing period. As a result, the solvent channels contract less during subsequent cooling and the crystal quality is more likely to improve. Juers and Matthew concluded that the failure of in situ cryoannealing may be an indication that the cryoprotectant concentration is too low and that annealing is most likely to be successful in situations where the cryoprotectant concentration is initially too high rather than too low. They suggest that annealing would thus only be beneficial in cases where cryoconditions had not been properly optimized.

For our studies, it should be noted that the cooling protocol for the glycerol kinase crystals was optimized extensively, so that the concentrations of cryoprotectants (ethylene glycol, glycerol, MPD, PEG 200, PEG 400) used were the minimum needed for glass formation at $100 \mathrm{~K}$, as indicated visually by the transparency of the solution without residual opacity. However, we later found that visual inspection is a necessary but not sufficient indicator of optimal cryoprotection, as further elaborated below. Additionally, a comparison of data collected at room temperature on crystals of glycerol kinase showed variations in mosaicities between 0.3 and $>0.8^{\circ}$. This suggests that inherent variations, possibly occurring during the crystal-growth stage, may be the major contributor to the overall disorder of the crystal rather than damage arising from the cooling protocol. This is further supported by the mosaicity values of cryocooled glycerol kinase crystals, which can be of the order of $0.3^{\circ}$, as indicated by the mosaicity given by the program $D E N Z O$. This idea is also substantiated by the study of copper nitrite reductase crystals, whose diffraction improved from 2.5 to $1.1 \AA$ after flash-annealing, whereas a room-temperature data set diffracted to $1.8 \AA$ (Ellis et al., 2002). The authors concluded that flash-annealing (in situ annealing) provided genuine improvement in crystal order and thus the resolution.

In this study, the measured reflection profiles and mosaicity values from glycerol kinase crystals were used to obtain a more precise model of the mosaic domain structure and composition of the crystals before and after flash-annealing. Reflection profiles were obtained via a fine $\varphi$-slicing method using highly monochromatic and parallel synchrotron radiation (Bellamy et al., 2000). The mosaicity, $\eta$, is deconvoluted from the measured angular width of each profile, $\varphi_{R}$, by applying corrections for the beam parameters and the Lorentz factor (Bellamy et al., 2000; Colapietro et al., 1992; Greenhough \& Helliwell, 1982). The number, shape and size of mosaic domains can be estimated from curve-fitting the complete reflection profile (Otálora et al., 1999).

\section{Methods}

\subsection{Crystal growth}

Crystals of Enterococcus casseliflavus glycerol kinase were obtained by the hanging-drop technique from a solution containing $29 \%$ polyethylene glycol $400,0.1 \mathrm{M}$ sodium acetate $\mathrm{pH}$ 4.5, $0.1 \mathrm{M}$ calcium acetate and 10\% glycerol. Crystals appeared in varying sizes from small needles to large rod-like crystals that were $0.7 \mathrm{~mm}$ long and $0.25 \mathrm{~mm}$ thick. The space group was determined as $P 2{ }_{1} 2{ }_{1} 2$, with a solvent content of $56 \%$, resulting in an occupancy of one dimer per asymmetric unit. The unit-cell parameters were $a=93.77, b=195.26$, $c=55.31 \AA$. This crystallization condition is different from that first found for the glycerol kinase crystals used in the original study (Yeh \& Hol, 1998) as are the space group and 
unit-cell parameters, although the solvent content remains very similar.

\subsection{Flash-annealing}

Annealing experiments were performed with a new device, the Cryo-Shutter, shown in Fig. 1, which was fabricated at Brookhaven National Laboratories. The Cryo-Shutter is a simple device that allows blocking for programmed amounts of time of the $100 \mathrm{~K}$ nitrogen stream that surrounds the sample. The shutter is controlled by EPICS (Experimental Physics and Industrial Control System) and activated by air pressure. The opening and closing time of the Cryo-Shutter is of the order of milliseconds, which is insignificant compared with the total annealing time. The main advantage of the shutter is that it allows a controlled instant re-cooling of the crystals and avoids significant turbulence induced by traditional annealing 'devices' such as a piece of hard thin plastic.

Although the glycerol kinase crystals can be mounted directly into cryoloops and flash-cooled in a $100 \mathrm{~K}$ nitrogen gas stream without apparent icing problems, it was found that this concentration of cryoprotectant (29\% PEG 400) was 'borderline' upon closer inspection. Apparently, at this concentration of PEG 400 the crystallization solution exhib-

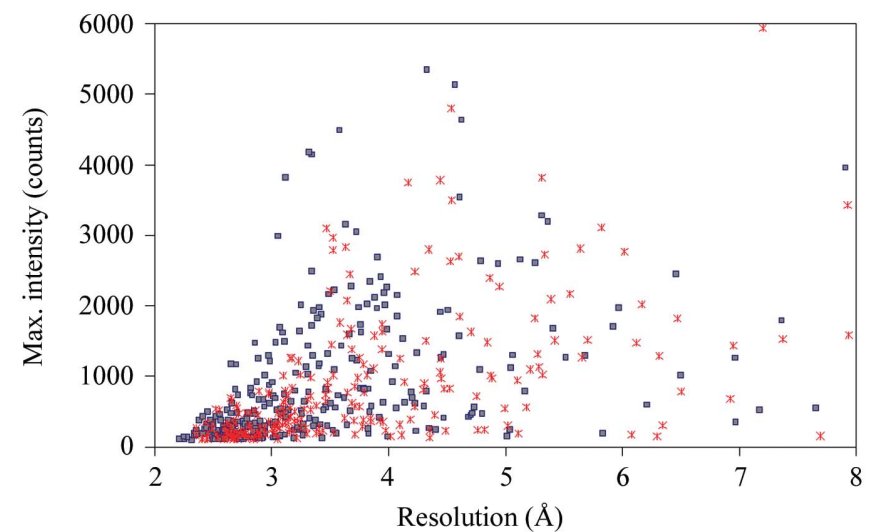

(a)

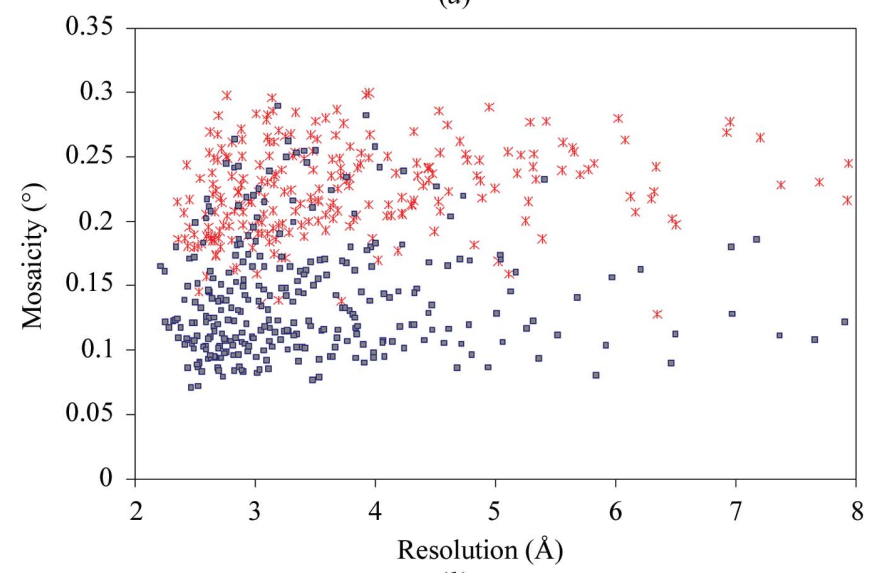

(b)

Figure 2

Crystal-quality comparison of glycerol kinase before (cryocooled, red crosses) and after flash-annealing (flash-annealed, blue diamonds) by plotting individual reflection $(a)$ mosaicity and $(b)$ peak count ('maximum intensity') against resolution.
Table 2

Diffraction and crystal mosaicity statistics for identical reflections in crystal xtl3.

\begin{tabular}{|c|c|c|c|c|}
\hline Crystal & 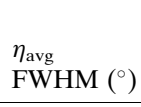 & $\begin{array}{l}\text { STDEV } \\
\eta_{\text {avg }}\left({ }^{\circ}\right)\end{array}$ & $\begin{array}{l}\text { Average max. } \\
\text { peak intensity } \\
\text { (counts) }\end{array}$ & $\begin{array}{l}\text { No. of reflection } \\
\text { analyzed }\end{array}$ \\
\hline \multicolumn{5}{|l|}{ Cryo versus annealed } \\
\hline cr_xtl3 & 0.276 & 0.063 & 789 & 79 \\
\hline an_xtl3 & 0.205 & 0.092 & 1016 & 79 \\
\hline Average difference $(\%)$ & -25.7 & 46.0 & 28.8 & \\
\hline
\end{tabular}

ited varying minute degrees of opacity upon cryocooling. The amount of opacity and icing, as indicated by a higher background of diffraction between 3.4 and $3.8 \AA$, was dependent on the age of the crystals and the mounting technique (i.e. the length of time a drop was open prior to mounting in a loop and cooling). We attributed this to dehydration, effectively increasing the concentration of PEG 400 to a concentration that prevented 'residual' icing, which correlated with the increased visual transparency of the cryocooled solution. We initially added an additional 4\% PEG 400 to the artificial mother liquor (29\% PEG 400, $0.1 M$ sodium acetate $\mathrm{pH} 4.5$, $0.1 M$ calcium acetate and $10 \%$ glycerol) and used this solution for mounting and cryocooling. However, we found that

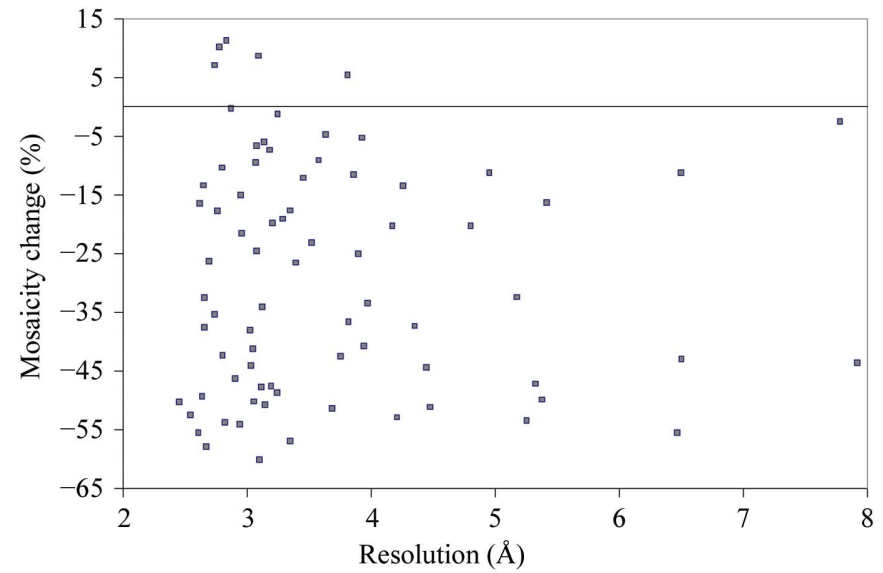

(a)

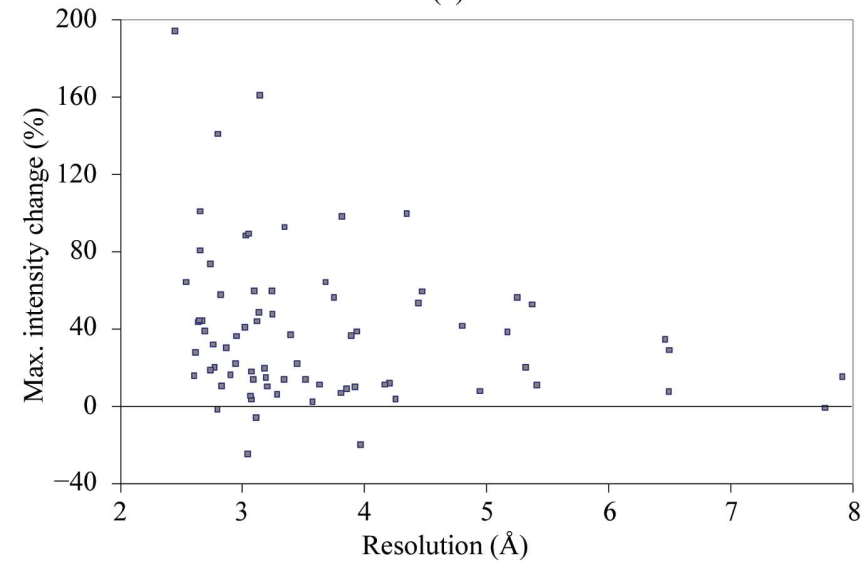

(b)

Figure 3

Crystal-quality comparison of glycerol kinase as a result of flashannealing by plotting identical reflection $(a)$ mosaicity change and $(b)$ peak-count change ('maximum intensity change') against resolution. 
we could grow the crystals in the presence of $33 \%$ PEG 400, which allowed us to mount the crystals and directly flash-cool them.

For flash-annealing, the Cryo-Shutter controller software was set to a $4 \mathrm{~s}$ annealing cycle. The annealing was repeated two times for each crystal as it was found from previous experiments that between two and three cool-thaw cycles improved the mosaicity and resolution, whereas greater numbers of cool-thaw cycles eventually degraded diffraction qualities. Thawing and re-cooling of the buffer and crystal were observed on a monitor during the flash-annealing process and the crystal remained transparent throughout the flashannealing process.

\subsection{Fine $\varphi$-sliced data collection and processing}

A highly parallel unfocused beam from a bending magnet on beamline X6a at the National Synchrotron Light Source (NSLS) was used to minimize beam divergence. The beam properties were calculated from the source size and slit apertures using the SHADOW software (Cerrina et al., 1998). The vertical and horizontal beam divergences were calculated to be 52.2 and $33.4 \mu \mathrm{rad}$, respectively, with primary slits set to $1.2 \times 0.8 \mathrm{~mm}$ just before the $\mathrm{Si}(111)$ channel-cut monochromator (Allaire et al., 2003) located at $7.8 \mathrm{~m}$ from the source. The secondary slits were set to $0.5 \times 0.5 \mathrm{~mm} 16.9 \mathrm{~m}$ from the source and $\Delta \lambda / \lambda$ was calculated to be $2 \times 10^{-4}$.

Data were collected with a Quantum-4 CCD detector (ADSC) using rotation-camera geometry as described previously (Bellamy et al., 2000; Borgstahl et al., 2001; Snell et al., 2001). To determine the crystal orientation, two orthogonal $3-6^{\circ}$ swaths of coarse data were collected $\left(\Delta \varphi=1^{\circ}\right.$ with $20 \mathrm{~s}$ exposure) and processed with MOSFLM (Powell, 1999). From each swath, a $1.5^{\circ}$ range was then selected and fine $\varphi$-sliced data corresponding to that range were collected for mosaicity measurements. Data were collected on three glycerol kinase crystals (denoted $\mathrm{xtl1}, \mathrm{xt} 2$ and $\mathrm{xtl} 3$ ). Each crystal was cryocooled directly in the nitrogen cryostream at $100 \mathrm{~K}$, data were collected (data denoted as 'cryo' and 'cr') and then the crystal was flash-annealed (data denoted as 'flash-

Figure 4 annealed' and 'FA'). The same data-collection parameters were used for each data set. Fine $\varphi$-sliced data were collected as stills spaced by $0.003^{\circ}$ with $2 \mathrm{~s}$ X-ray exposures and a total of 500 frames were collected for each crystal at $100 \mathrm{~K}$. The crystal-to-detector distance was $225 \mathrm{~mm}$ and the resolution at the edge of the detector was 2.3 and $1.8 \AA$ at the corners. The wavelength was $1.033 \AA$ and the beam was collimated to $0.141 \mathrm{~mm}$ diameter. Each data set took less than $20 \mathrm{~min}$ per crystal, therefore decay in the beam over time was ignored in the analysis. Crystal decay, as indicated by the DENZO scale factor per frame, was negligible. The fine $\varphi$-sliced data were
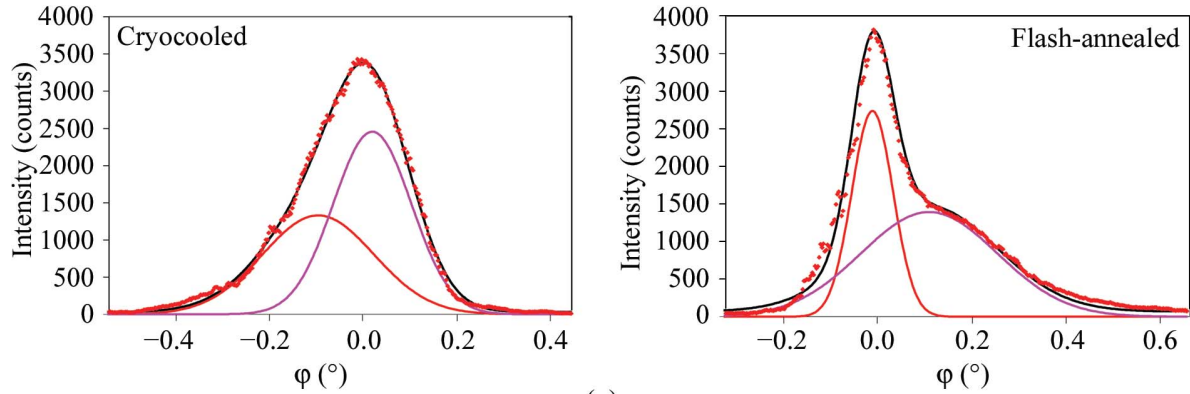

(a)
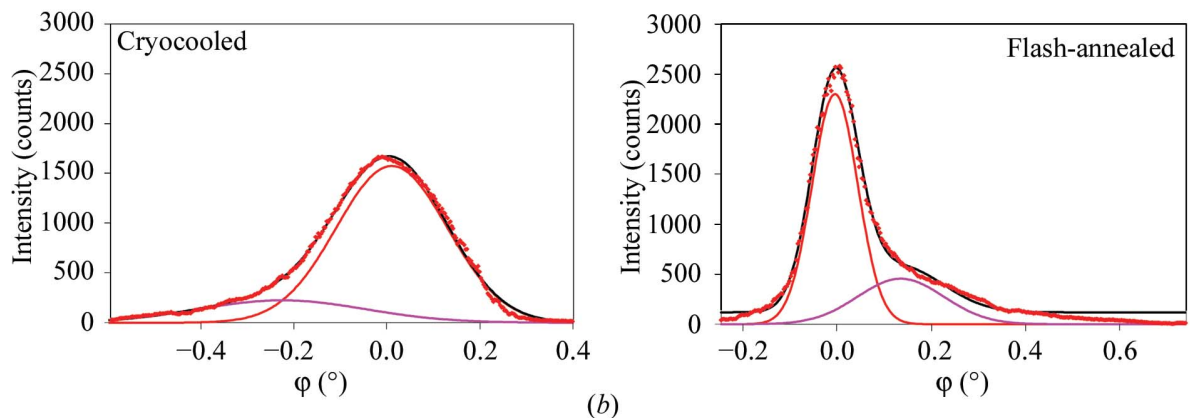

(b)
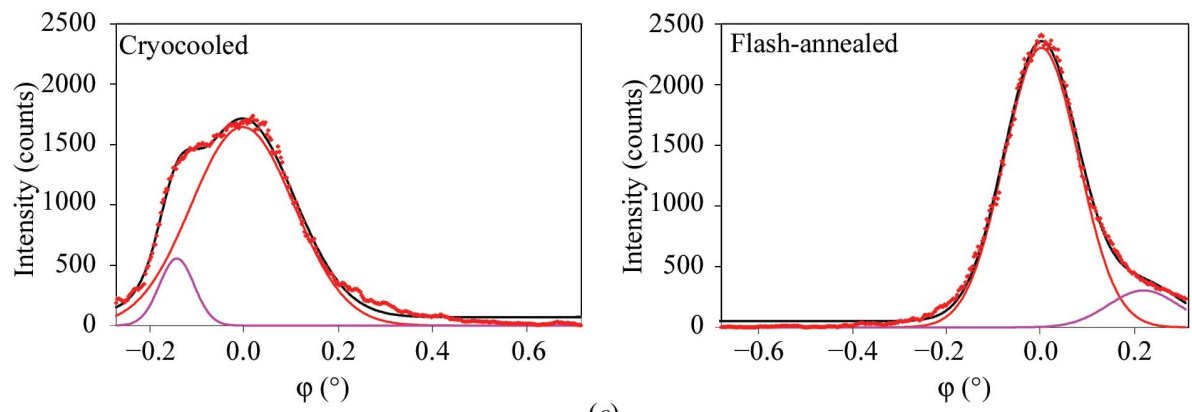

(c)
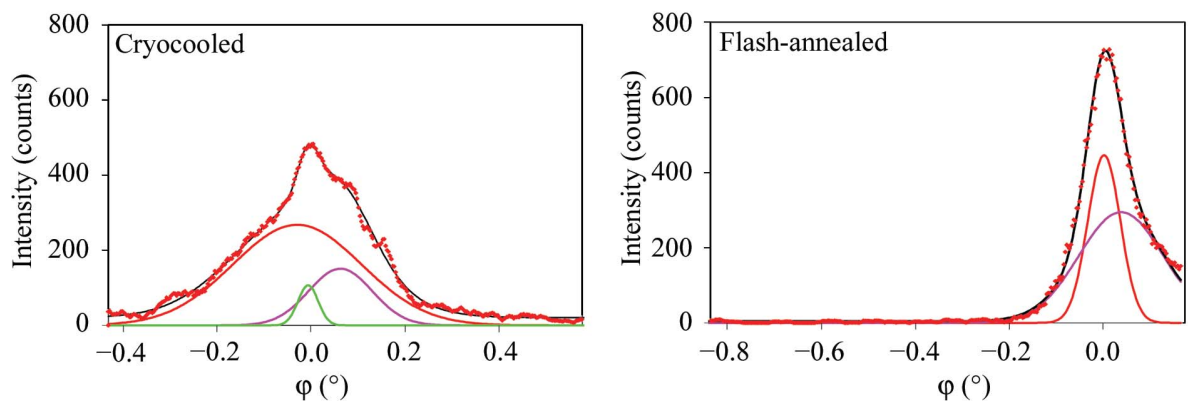

(d)

Seven representative reflection profiles out of 79 available identical reflections for crystals xtl3 (see Table 3). The red diamonds mark the measured individual raw data points. The black graph represents a summation of the fitted individual Gaussians (red, purple and green). 
Table 3

Analysis of mosaic domain structure in xtl3 crystal.

\begin{tabular}{|c|c|c|c|c|c|c|c|c|}
\hline & \multicolumn{2}{|l|}{ Peak } & \multicolumn{2}{|c|}{ Gaussian 1} & \multicolumn{2}{|c|}{ Gaussian 2} & \multirow[b]{2}{*}{$\begin{array}{l}\text { Separation } \\
\varphi 1-\varphi 2\left(^{\circ}\right)\end{array}$} & \multirow[b]{2}{*}{$\begin{array}{l}\Delta \text { Are } \\
(\%)\end{array}$} \\
\hline & $\begin{array}{l}\text { FWHM } \\
\left({ }^{\circ}\right)\end{array}$ & $\begin{array}{l}I_{\text {MAX }} \\
\text { (counts) }\end{array}$ & $\begin{array}{l}\text { FWHM } \\
\left({ }^{\circ}\right)\end{array}$ & $\begin{array}{l}I_{\text {MAX }} \\
\text { (counts) }\end{array}$ & $\begin{array}{l}\text { FWHM } \\
\left(^{\circ}\right)\end{array}$ & $\begin{array}{l}I_{\text {MAX }} \\
\text { (counts) }\end{array}$ & & \\
\hline \multicolumn{9}{|c|}{$h=1, k=-5, l=-22$} \\
\hline Cryocooled & 0.252 & 3428 & 0.283 & 1329 & 0.198 & 2453 & 0.117 & \\
\hline Flash-annealed & 0.129 & 3954 & 0.105 & 2535 & 0.346 & 1391 & 0.121 & -8.5 \\
\hline \multicolumn{9}{|l|}{$h=2, k=10, l=30$} \\
\hline Cryocooled & 0.305 & 1666 & 0.282 & 1573 & 0.425 & 224 & 0.237 & \\
\hline Flash-annealed & 0.122 & 2607 & 0.116 & 2267 & 0.218 & 454 & 0.141 & -11.7 \\
\hline \multicolumn{9}{|c|}{$h=4, k=13, l=39$} \\
\hline Cryocooled & 0.303 & 1738 & 0.261 & 1645 & 0.088 & 555 & 0.142 & \\
\hline Flash-annealed & 0.182 & 2409 & 0.187 & 2302 & 0.177 & 303 & 0.219 & -3.9 \\
\hline \multicolumn{9}{|c|}{$h=-2, k=21, l=53$} \\
\hline Cryocooled & 0.241 & 483 & 0.319 & 268 & 0.157 & 151 & 0.091 & \\
\hline Flash-annealed & 0.105 & 762 & 0.209 & 294 & 0.081 & 446 & 0.037 & -13.1 \\
\hline \multicolumn{9}{|c|}{$h=3, k=-11, l=-69$} \\
\hline Cryocooled & 0.215 & 648 & 0.255 & 286 & 0.163 & 407 & 0.098 & \\
\hline Flash-annealed & 0.131 & 1171 & 0.236 & 252 & 0.116 & 1017 & 0.130 & +50.8 \\
\hline \multicolumn{9}{|c|}{$h=2, k=-9, l=-49$} \\
\hline Cryocooled & 0.260 & 1383 & 0.170 & 786 & 0.249 & 865 & 0.110 & \\
\hline Flash-annealed & 0.116 & 2272 & 0.207 & 489 & 0.110 & 1931 & 0.116 & -3.5 \\
\hline \multicolumn{9}{|l|}{$h=7, k=13, l=40$} \\
\hline Cryocooled & 0.391 & 1202 & 0.122 & 281 & 0.503 & 563 & 0.274 & \\
\hline Flash-annealed & 0.335 & 1129 & 0.309 & 1057 & 0.415 & 186 & 0.242 & -6.2 \\
\hline
\end{tabular}
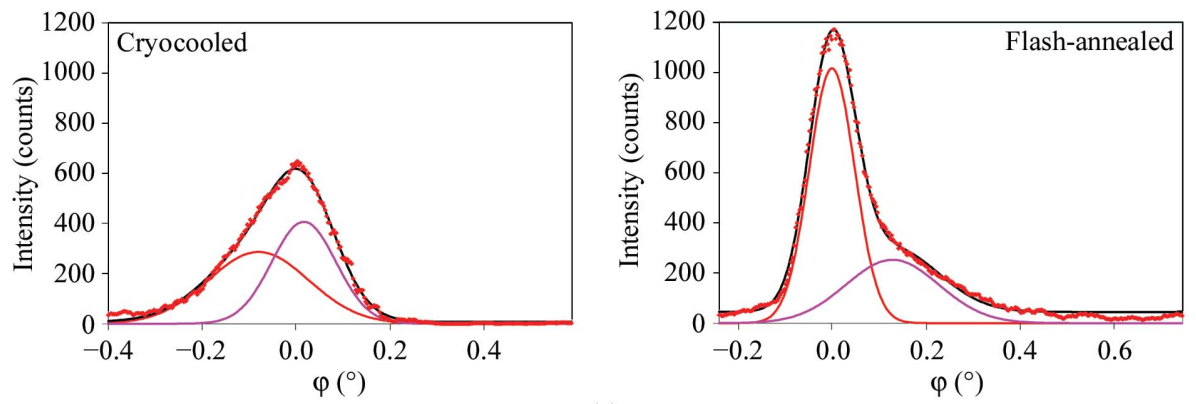

(e)
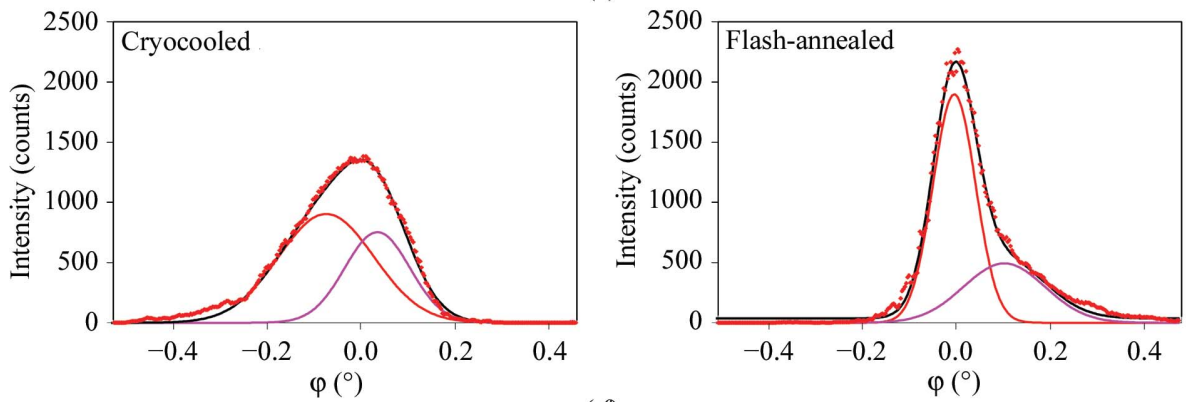

$(f)$
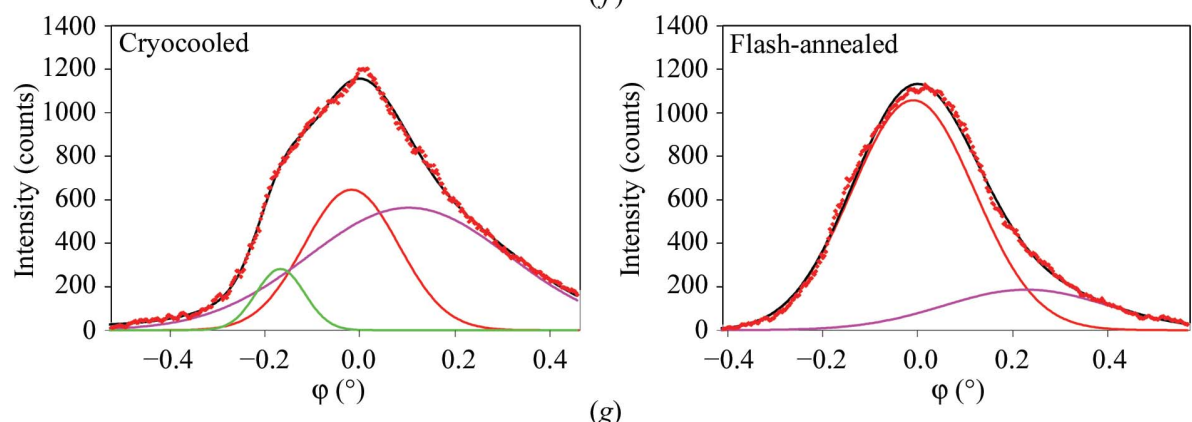

(g)

Figure 4 (continued) processed and analyzed using $B E A M$ ish 2.0 (Lovelace et al., 2000). The program BEAM-ish deconvolutes the reflection mosaicity, $\eta$, using the measured reflection full-width at halfmaximum, $\varphi_{\mathrm{R}}$, as described by Bellamy et al. (2000) and Helliwell (1988). To reduce noise, the data points were averaged over $0.006^{\circ}$, with two slicing events in a $0.003^{\circ}$ window. In order to be accepted for profile analysis the reflections had to have $I_{\max }>100$ and $I / \sigma(I)>5$. Gaussian curves were fit to the reflection profiles using a genetic algorithm (Wormington et al., 1999) and the inter-process communication feature of BEAM-ish 2.0 was used to search for identical reflections across multiple data sets (Lovelace et al., 2000).

\section{Results and discussion}

\subsection{Effects of flash-annealing on mosaicity, peak intensity and resolution}

Annealing approaches have been proposed by several groups (Yeh \& Hol, 1998; Bunick et al., 1998; Harp et al., 1998, 1999; Kriminski et al., 2002) to overcome the disadvantage of increased mosaicity as a result of cryocooling or to generally improve data quality. In the current study, mosaic domain theory was applied to the interpretation of the reflection profiles (Vahedi-Faridi et al., 2003) measured from the glycerol kinase crystals. A total of three glycerol kinase crystals (xtl1, xtl2 and xtl3) were examined to study the effects of flashannealing on crystal quality. 500 images each with an oscillation angle of $0.003^{\circ}$ were collected before (denoted as 'cryo' and 'cr') and after annealing (denoted as 'flash-annealed' and 'FA') to obtain reflection profiles and to deconvolute reflection mosaicity and peak-intensity values. Mosaicity data were measured from the full-width at half-maximum (FWHM) and full-width at quarter-maximum (FWQM) of reflection profiles. Table 1 shows a summary of the averaged diffraction statistics for each of the three crystals examined. Flash-annealing resulted in 
a noticeable overall decrease of the averaged reflection mosaicities, ranging from $26.3 \%$ (xt11) to a decrease of $38.2 \%$ for xt13 (see Fig. 2a). The averaged maximum pixel count for all reflections decreased slightly, as indicated by the change in peak area. However, the overall decrease in maximum intensities can be attributed to the fact that a filter was imposed for reflections to be included in the statistical analysis $\left[I / \sigma(I)>5\right.$ and $I_{\mathrm{MAX}}>100$; see $\left.\$ 2.3\right]$. The improvement in crystal quality as a result of flash-annealing allowed many more reflections to pass this filter (e.g. an increase of 48 reflections for xtl3). Most of these additional reflections have a peak count slightly above 100 , thus contributing to the small overall decrease in the averaged values. The peak count is also referred to as 'maximum intensity' in the figures and is the count at the peak of the rocking curve. Fig. 2(b) shows a plot of the maximum peak intensities as a function of resolution for crystal xtl3. The plot shows 'annealed' low-intensity reflections at a resolution greater than $2.5 \AA$, showing an increase in diffractive resolution as a result of flash-annealing.

Detailed comparisons of orientation matrices and observed reflection profiles showed that crystals xt11 and xtl2 underwent a noticeable shift in position within the cryoloop during the flash-annealing process. As a result, identical reflections were not recorded for these crystals within the two measured $1.5^{\circ}$ swaths. Crystal xtl3, however, moved to a far lesser degree, allowing the comparison of 79 identical reflections before and after flash-annealing (see Table 2). The averaged mosaicity decrease for all 79 reflections is $25.7 \%$. We integrated the peak areas of seven reflections to quantify the change before and after flash-annealing (Table 3 , column $\Delta$ Area). For the seven reflections, all but one exhibited a small decrease in peak areas; however, for the one reflection $(3,-11,-69)$ whose peak area increased, the gain after annealing was significant $(+50.8 \%)$. Contrary to the averaged value for all reflections, the averaged maximum peak intensity counts in the most intense pixels for all identical reflections actually increased by $28.8 \%$. The increase of $46 \%$ in the standard deviation for the reflection mosaicities indicates that although reflection profiles become narrower overall as a result of flash-annealing, the mosaicity distribution for individual reflection increases overall. This fact is evident in Fig. 2(a), showing annealed reflections with smaller mosaicities but wider mosaicity distribution as a function of resolution. Figs. $3(a)$ and $3(b)$ show a plot of the mosaicity and peak-count change ('maximum intensity change', in percent) as a function of resolution. Approximately $93 \%$ of all observed identical reflections showed a decrease in their reflection mosaicities along with an increase in the corresponding peak intensities.

\subsection{Effect of flash-annealing on reflection profiles and domain structure}

The availability of a large number of identical reflections allows detailed characterization of the annealing effects on the reflection profiles and domain structure. Fig. 4 shows 14 representative reflection profiles corresponding to seven identical reflections from crystal xtl3. In general, the cryo- cooled reflection profiles had a similar number of mosaic domains as the 'annealed' reflections (Figs. 4). All of the 'annealed' peak profiles were described by two Gaussian curves. Thus, 'annealed' reflections still retain a dual-domain conformation with a small shoulder adjacent to the main peak. Most cryocooled profiles were also described by two Gaussians, with a few exceptions which were fitted with an additional third small Gaussian (Figs. $4 d$ and $4 g$ ). The most noticeable effect of flash-annealing is the gain in maximum peak height along with a decrease in peak width for one fitted Gaussian at the expense of the second (Table 3). These plots suggest that the decreased mosaicity can arise from improved alignment of non-resolvable domains or a decrease in the variation of lattice spacings. Overall peak profiles are much more homogeneous and symmetrical. As a result of the enhancement of a single domain after flash-annealing, the signal-to-noise ratio of the data is improved by increasing the peak height of the reflections, making the weaker and higher resolution data easier to measure.

Another mechanism for decreased mosaicity is through improved domain misalignment and this may be exhibited through decreased peak separation of the Gaussian profiles of reflections after annealing. Our results indicate that two are approximately the same, three are reduced and two are increased (Table 3). Consequently, within the subset of reflections that we analyzed there appears to be a random distribution, although a trend may possibly be detected with a larger database for analysis.

\section{Conclusions}

Overall, the mosaic domain structure was changed as a result of flash-annealing, resulting in clearly improved profile shapes for the 'flash-annealed' crystals. Flash-annealing significantly decreased the mosaicity of glycerol kinase crystals. The sharpening of individual domains suggests that there is a decrease in unit-cell variation within the domain. Reflections had improved signal-to-noise ratios as a result of flash-annealing, thus contributing significantly to the detection and measurement of higher resolution data.

We thank Anthony Lenhard and Zhihiang Yin of the National Synchrotron Light Source, Brookhaven National Laboratory for their assistance. This work was supported by NIH (GM/AI66466) and March of Dimes Foundation (5-FY00-564) to JIY. The National Synchrotron Light Source, Brookhaven National Laboratory is supported by the US Department, and X6A is funded by NIH/NIGMS under agreement Y1 GM-0080 Energy under contract No. DEAC02-98CH10886.

\section{References}

Allaire, M., Aslantas, M., Berntson, A., Berman, L., Cheung, S., Clay, B., Greene, R., Jakoncic, J., Johnson, E., Kao, C. C., Lenhard, A., Pjerov, S., Siddons, D. P., Stober, W., Venkatagiriyappa, V., Yin, Z. \& Stojanoff, V. (2003). Synchrotron Radiat. News, 16, 20-25. 
Bellamy, H. D., Snell, E. H., Lovelace, J., Pokross, M. \& Borgstahl, G. E. O. (2000). Acta Cryst. D56, 986-995.

Boggon, T. J., Helliwell, J. R., Judge, R. A., Olczak, A., Siddons, D. P., Snell, E. H. \& Stojanoff, V. (2000). Acta Cryst. D56, 868-880.

Borgstahl, G. E. O., Vahedi-Faridi, A., Lovelace, J., Bellamy, H. D. \& Snell, E. H. (2001). Acta Cryst. D57, 1204-1207.

Bunick, G., Harp, J., Timm, D. \& Hanson, L. (1998). Rigaku J. 15, 6-13.

Cerrina, F., Lai, B., Chapman, K., Welnak, C. \& Runkle, P. (1998). SHADOW v.2.3.3. A System for the Study of Optical Sytems. University of Wisconsin-Madison, USA.

Colapietro, M., Cappuccio, G., Marciante, C., Pifferi, A., Spagna, R. \& Helliwell, J. R. (1992). J. Appl. Cryst. 25, 192-194.

Darwin, C. G. (1922). Philos. Mag. 43, 800-829.

Ellis, M. J., Antonyuk, S. \& Hasnain, S. S. (2002). Acta Cryst. D58, 456-458.

Garman, E. F. \& Schneider, T. R. (1997). J. Appl. Cryst. 30, 211-237.

Greenhough, T. J. \& Helliwell, J. R. (1982). J. Appl. Cryst. 15, 338351.

Harp, J. M., Hanson, B. L., Timm, D. E. \& Bunick, G. J. (1999). Acta Cryst. D55, 1329-1334.

Harp, J. M., Timm, D. E. \& Bunick, G. J. (1998). Acta Cryst. D54, 622628.

Helliwell, J. R. (1988). J. Cryst. Growth, 90, 259-272.

Juers, D. H. \& Matthews, B. W. (2004). Acta Cryst. D60, 412-421.

Kriminski, S., Caylor, C. L., Nonato, M. C., Finkelstein, K. D. \& Thorne, R. E. (2002). Acta Cryst. D58, 459-471.

Lovelace, J., Snell, E. H., Pokross, M., Arvai, A. S., Nielsen, C., Xuong, N., Bellamy, H. D. \& Borgstahl, G. E. O. (2000). J. Appl. Cryst. 33, 1187-1188.
Mitchell, E. P. \& Garman, E. F. (1994). J. Appl. Cryst. 27, 10701074.

Nave, C. (1998). Acta Cryst. D54, 848-853.

Otálora, F., Capelle, B., Ducruix, A. \& García-Ruiz, J. M. (1999). Acta Cryst. D55, 644-649.

Otwinowski, Z. (2005). Personal communication.

Otwinowski, Z. \& Minor, W. (2001). International Tables for Crystallography, Vol. F, edited by M. G. Rossmann \& E. Arnold, ch. 11.4. Dordrecht: Kluwer Academic Publishers.

Parak, F., Hartmann, H., Aumann, K. D., Reuscher, H., Rennekamp, G., Bartunik, H. \& Steigemann, W. (1987). Eur. Biophys. J. 15, 237249.

Parkin, S. \& Hope, H. (2003). Acta Cryst. D59, 2228-2236.

Petsko, G. (1975). J. Mol. Biol. 96, 381-392.

Powell, H. R. (1999). Acta Cryst. D55, 1690-1695.

Rodgers, D. W. (1994). Structure, 2, 1135-1140.

Rodgers, D. W. (1997). Methods Enzymol. 276, 183-202.

Samygina, V. R., Antonyuk, S. V., Lamzin, V. S. \& Popov, A. N. (2000). Acta Cryst. D56, 595-603.

Snell, E. H., Judge, R. A., Crawford, L., Forsythe, E. L., Pusey, M. L., Sportiello, M., Todd, P., Bellamy, H., Lovelace, J., Cassanto, J. M. \& Borgstahl, G. E. O. (2001). Cryst. Growth Des. 1, 151-158.

Teng, T.-Y. \& Moffat, K. (1998). J. Appl. Cryst. 31, 252-257.

Vahedi-Faridi, A., Lovelace, J., Bellamy, H., Snell, E. \& Borgstahl, G. E. O. (2003). Acta Cryst. D59, 2169-2182.

Wormington, M., Panaccione, C., Matney, K. M. \& Bowen, D. K. (1999). Philos. Trans. R. Soc. London Ser. A, 357, 28272848.

Yeh, J. I. \& Hol, W. G. J. (1998). Acta Cryst. D54, 479480. 